2005-08-31

\title{
Studies in the Dynamics of Economic Systems
}

Christophe G. Giraud-Carrier

cgc@cs.byu.edu

Kevin Seppi

Brigham Young University, kseppi@byu.edu

Nghia Tran

Sean C. Warnick

W. Samuel Weyerman

See next page for additional authors

Follow this and additional works at: https://scholarsarchive.byu.edu/facpub

Part of the Computer Sciences Commons

\section{Original Publication Citation}

N. Tran, W. Weyerman, C. Giraud-Carrier, K. Seppi, S. Warnick, and R. Johnson. "Studies in the Dynamics of Economic Systems." In Proceedings of the IEEE Conference on Control Applications, 25.

\section{BYU ScholarsArchive Citation}

Giraud-Carrier, Christophe G.; Seppi, Kevin; Tran, Nghia; Warnick, Sean C.; Weyerman, W. Samuel; and Johnson, R., "Studies in the Dynamics of Economic Systems" (2005). Faculty Publications. 1006. https://scholarsarchive.byu.edu/facpub/1006 accepted for inclusion in Faculty Publications by an authorized administrator of BYU ScholarsArchive. For more information, please contact ellen_amatangelo@byu.edu. 


\section{Authors}

Christophe G. Giraud-Carrier, Kevin Seppi, Nghia Tran, Sean C. Warnick, W. Samuel Weyerman, and R. Johnson 


\title{
Studies in the Dynamics of Economic Systems
}

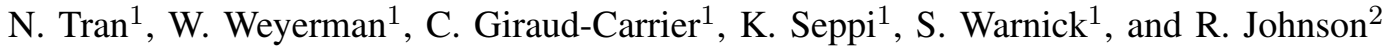 \\ ${ }^{1}$ Information Dynamics and Intelligent Systems Group \\ Computer Science Department, Brigham Young University, Provo, UT 84602 \\ ${ }^{2}$ Charles River Associates, Inc. \\ Salt Lake City, UT 84101
}

\begin{abstract}
This paper demonstrates the utility of systems and control theory in the analysis of economic systems. Two applications demonstrate how the analysis of simple dynamic models sheds light on important practical problems. The first problem considers the design of a retail laboratory, where the small gain theorem enables the falsification of pricing policies. The second problem explores industrial organization using the equilibria of profit-maximizing dynamics to quantify the percentage of a firm's profits due strictly to the cooperative effects among its products. This "Value of Cooperation" suggests an important measure for both organizational and antitrust applications.
\end{abstract}

\section{INTRODUCTION}

In today's society, economic systems are among the most heavily instrumented of any engineered system. Point-ofsale data-capturing mechanisms, such as scanners, are ubiquitous, and businesses invest heavily in data warehousing and analysis technologies. With the advent of the internet, it is not uncommon for firms to warehouse terabytes of data. Various disciplines, such as statistics, data mining, machine learning, and operations research have postured to help managers transform this data into useful decisions. In spite of these efforts, control theoretic approaches to the problem still add unique value by emphasizing dynamic behavior and the effects of feedback.

This paper demonstrates the point by exploring the implications of simple dynamic models of economic phenomenon. Section II. highlights the first example, discussing recent work in the design of a live retail laboratory at Brigham Young University. Retail is modeled as a feedback process. Optimal control formulations of various pricing mechanisms have recently gained renewed popularity in the field of Revenue Management [8], but little work has studied the verification of such mechanisms. Our work on the design of a retial laboratory reveals the importance of robust control formulations for yielding pricing strategies capable of being conclusively invalidated.

Section III. develops an entirely different view of market dynamics emphasizing the competitive interaction between firms and their subsequent industrial organization. Here

This work is supported by grants from the Rollins Center for eBusiness, the Office of Research and Creative Activities at BYU, the BYU Bookstore, and Sandia National Laboratories. Please direct all comments and questions to Sean Warnick at sean@cs.byu.edu . we show that a game-theoretic measure on the value of cooperation emerges naturally by studying how equilibria of the market system change under different coalition models. The point is made on a simple example.

In both of these cases, simple dynamic models facilitate insight into the nature of economic behavior. Although economics and control have an established history, it is our view that economic systems continue to provide a rich application domain for control theory to contribute to a truly interdisciplinary area [4], [9].

\section{Design of a Retail Laboratory}

\section{A. Retail as a Feedback Process}

We begin our discussion with the idea of a firm. Our firm will begin its life with a fixed amount of capital, $C(0)$, (or credit available, but our assumption is that there is a fixed bound on the purchase power available to the firm), and a list of $n$ wholesale goods it can choose to purchase. There may be many potential suppliers of these goods, so the actual information the firm receives is a matrix of wholesale prices (effective costs) we denote as a $p \times n$ matrix $P_{W}(k)$, where $k=0,1,2, \ldots$ is the number of days since the firm began operation. Thus, $P_{W}(k)$ are the prices the firm receives on the morning of day $k$, and we assume they will not change until the next morning.

The firm purchases wholesale goods on day $k$ at price $P_{W}(k)$ by specifying a matrix $X_{W}(k)$, which identifies how many of the $n$ goods from each of the $p$ suppliers the firm will purchase at the current price, $P_{W}(k)$. Of course, the firm has limits on its spending characterized by the constraint that $\sum_{i, j} x_{w(i, j)} p_{w(i, j)} \leq C(k)$, where $x_{w(i, j)}$ and $p_{w(i, j)}$ are the $(i, j)$ th elements of $X_{W}(k)$ and $P_{W}(k)$, respectively.

The firm then makes offers to its retail market for a price of its choosing. Since the firm may offer different effective prices to its $m$ customers (or different store locations, or other type of channel), say through coupons or special offers, etc., the pricing decision of the firm is represented by a $m \times n$ matrix $P_{R}(k)$, which means the prices available to the $m$ retail customers (or channels, or segments) of the $n$ goods on the morning of day $k$. Note that goods that the firm has not purchased wholesale are listed as available for very high prices. 


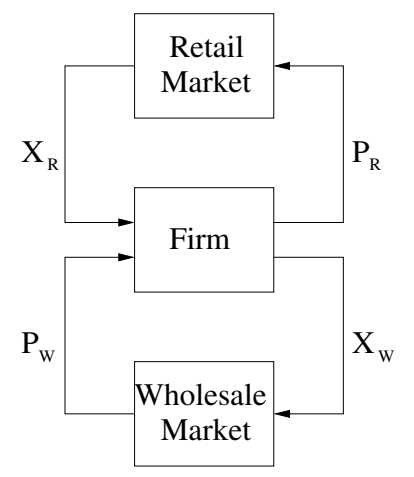

Fig. 1. A retail firm interacts with wholesale and retail markets.
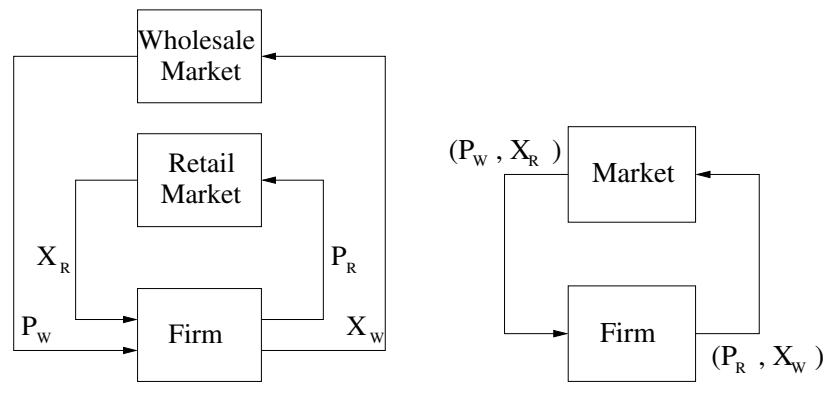

Fig. 2. By rearranging Figure 1 (left), we note that the distinction between a retail and wholesale market simply imposes structure on the more general feedback relationship (right).

The retail market then responds with purchases throughout day $k$ characterized by a matrix $X_{R}(k)$. This $m \times n$ matrix describes how much of each of the $n$ goods each of the $m$ retail customers or segments purchased that day. At this point, money from the day's transactions are collected, and the next morning's available capital becomes $C(k+1)=C(k)-\sum_{i, j} x_{w(i, j)} p_{w(i, j)}+\sum_{i, j} x_{R(i, j)} p_{R(i, j)}$.

Although real prices are quantized by the fundamental monetary unit, and the unavailability of a good may be considered ontologically different than associating a very high price with the good, we will ignore these details for simplicity and consider that $P_{W}(k) \in \mathbb{R}^{p \times n}$ and $P_{R}(k) \in \mathbb{R}^{m \times n}$. We thus define the firm as an operator $F:\left(\mathbb{R}^{m \times n} \times \mathbb{R}^{p \times n}\right) \rightarrow\left(\mathbb{R}^{m \times n} \times \mathbb{R}^{p \times n}\right)$, its wholesale market as an operator $M_{W}: \mathbb{R}^{p \times n} \rightarrow \mathbb{R}^{p \times n}$, and its retail market as an operator $M_{R}: \mathbb{R}^{m \times n} \rightarrow \mathbb{R}^{m \times n}$, which yields the picture shown in Figure 1. More generally, we could consider cases where the wholesale and retail markets are interrelated, yielding a combined market operator $M$ : $\left(\mathbb{R}^{m \times n} \times \mathbb{R}^{p \times n}\right) \rightarrow\left(\mathbb{R}^{m \times n} \times \mathbb{R}^{p \times n}\right)$. This results in a very general feedback structure as shown in Figure 2, where $C$ is an internal state of the firm. We assume that such interconnection is well-posed, in that solutions of the system $u$ and $y$ exist and are unique.

Notice that in this view of retail, a firm's interaction with the market is to buy and sell. Coupons, markdowns, special promotions, advertising, etc. are all modeled as equivalent modes of communicating offers to the firm's customers. Likewise, all modes of communication with a firm's suppliers are considered equivalent. Although this may not be precise for real economic systems, it is a reasonable simplification for the purposes of our analysis.

Furthermore, note that usual classifications of the firm as a price-setter or price-taker, or of the market as being competitive, oligopolistic, or monopolistic are unnecessary here. The firm makes its procurement and pricing decisions with the best information it has at any particular time, and information about whether it is operating in a competitive environment or has some special market power will be reflected in how the market responds.

\section{B. Validation of a Learned System}

With this view of the feedback process, where all market influences and disturbances have been lumped together into one operator $M: \mathcal{U} \rightarrow \mathcal{Y}$, we want to explore fundamental limits on validating a learning process. We may suppose that $M$ has a state space realization of the form

$$
\begin{gathered}
x(k+1)=f(x(k), u(k), k) \quad x(0)=x_{o} \\
y(k)=g(x(k), u(k), k)
\end{gathered}
$$

where $u$ is a vector of size $p \times n \times m \times n$ (the number of distinct decisions the firm can make at time $k$ ), $x$ is a presumably very large vector of internal market states (that we, nevertheless, assume to be finite), and $y$ a vector of market observations (same length as $u$ ). We assume that the firm has no special information about its market, and thus only knows $[u(k), y(k)]$ over a finite duration $k=0,1,2, \ldots, t$. In particular, the firm does not observe the internal market states $x$, nor does it have complete information of $f$ or $g$.

Once in operation, however, an intelligent firm will begin to consider its observations $[u(k), y(k)], k=0,1, \ldots, t$ and use them to develop some understanding of how its market behaves. It may accomplish this by hiring experts who retain some partial information of the market dynamics $f$ and $g$, or by employing novel learning processes that analyze its data in effective ways. However the firm operates, though, it will, explicitly or implicitly, develop a model, or theory, of how the market behaves and attempt to use this understanding to its advantage.

Assuming that the firm has explicitly developed a model of its market, consider the model as an operator $T: \mathcal{V} \subset$ $\mathcal{U} \rightarrow \mathcal{Y}$. That is, the understanding a firm obtains about its market enables it to establish expectations about what it will observe for at least some of the actions it might take $(u \in \mathcal{V})$. Regardless of how the firm developed its particular model $T$, however, it will need to determine how well the model approximates the true market before it will have the confidence to widely use the model to develop pricing or other policies. Restricting the market to the situations where the model applies, one approach the firm could take would be to measure some notion of distance between $M$ and $T$. 
Ideally, one would measure this error using the gain, or induced norm, of an operator, given as

$$
\|E\|=\sup _{u \neq 0} \frac{|E u|}{|u|}
$$

where $|\cdot|$ indicates an appropriate signal norm, and $E=$ $M-T$ is the prediction error. In practice, however, this quantity is difficult to compute. The first challenge arises from the fact that each signal $u$ or $y$ is a time function of infinite duration. Next, a search over all possible inputs $u \in \mathcal{V}$ to find the supremum is impractical, especially when most firms typically have market response data for only a few different pricing functions (e.g. often only the "normal" price followed by a sale price).

Finally, even if these other challenges could be resolved, just measuring the prediction error for a single, particular pricing function for even a limited duration is a difficult task. Although we may know how many widgets actually sold for three months in response to a particular, even a constant price, it is difficult to generate a comparable response from a market model because it is unclear how to set the model's initial conditions. If the market is assumed to be asymptotically stable, then the effects of an incorrect choice of initial conditions eventually would become negligible. Nevertheless, it would be unclear how long one should wait for this to occur. Moreover, the assumption of market stability in the first place seems questionable in an application domain bent on unbounded growth, ever-present competition, and ubiquitous "business cycles".

These difficulties cause various modes of approximate validation heuristics to be used in practice. The most sophisticated of these use cross validation techniques to attempt to approximate a measure of the modeling error. Ultimately, this approach of trying to validate a learned system by measuring the model error is attractive in that the process can be conducted entirely from cached data. As a result, the data warehousing industry has emerged, offering firms the chance to store terabytes of data to drive both learning and the subsequent validation processes.

Nevertheless, the link between these practical heuristics and real validation is not firmly established. An alternate validation process addresses the above difficulties, but at the cost of demanding more involvement from the firm in the scientific process than merely caching its data.

\section{The Retail Laboratory and the Small Gain Theorem}

Instead of trying to measure a comprehensive error of a market model, another approach to validation focuses on whether the proposed model is sufficient for an intended purpose. This approach uses a given model to design feedback policies that accomplish a specified objective. The effects of these policies on the true market are then compared with the results expected from the model, and the small gain theorem is employed to falsify models that are insufficient for the stated objective.
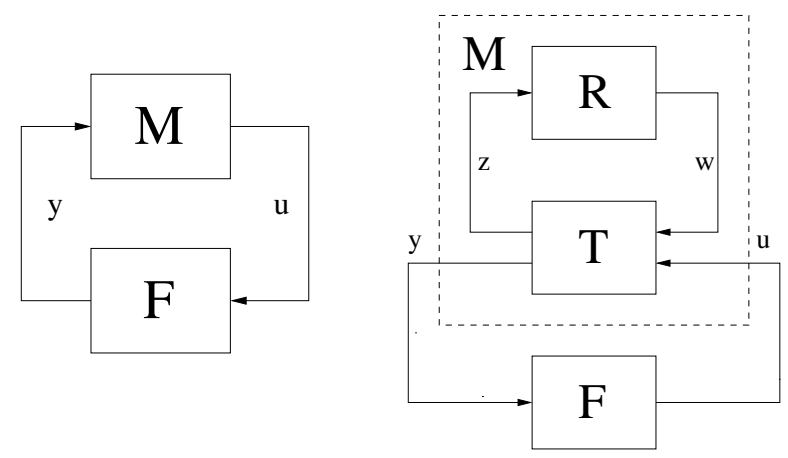

Fig. 3. The operator $M$ can be factored as the feedback interconnection of $R$ and $T$.

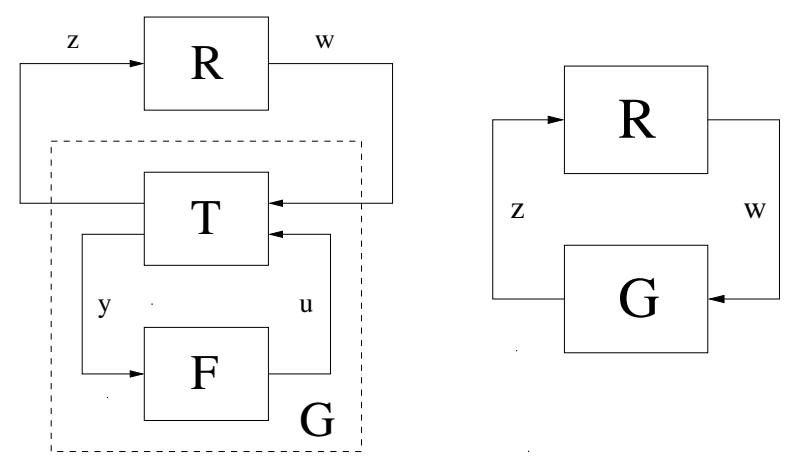

Fig. 4. Regrouping operators, the small gain theorem guarantees that $F$ stabilizes $M$ provided $\|G\|\|R\|<1$.

In particular, the true market $M$ can be factored into the feedback interconnection of a theory of the market $T$ with the unknown remainder $R$. This feedback factorization often allows an unstable operator $M$ to be decomposed into the feedback interconnection of two stable operators $R$ and $T$. Thus, although the conditions of the small gain theorem require that the unknown part of the market, $R$, be stable, this is a weaker condition than demanding that the market itself, $M$, be stable.

The novelty of the approach arises when we consider actually implementing a specific policy $F$ that translates observations from the market, $y$ into specific actions $u$. Although $F$ may be designed only with information of $T$, the small gain theorem says it will stabilize the real market $M$ provided $\|R\|\|G\|<1$, where $G$ is the feedback interconnection of $F$ and $T$. Note that we expect $G$ to be stable since we design $F$ such that it stabilizes $T$.

Thus, the scientist can test the quality of a given market theory $T$ by designing a policy $F$ that robustly and asymptotically stabilizes it with a desired rate of convergence. Analysis or simulation of this "ideal" closed-loop system, $G$, then indicates how long it will take the feedback interconnection of $F$ on any system $M$ "close" to $T$ (in the sense that $\|R\|$ is small enough) to stabilize. This suggests a finite duration experiment that can be tested empirically by implementing the policy $F$ "live" on the true market $M$. Observing the closed-loop results over the required duration 
either results in success, meaning the closed-loop market is behaving as designed (at least so far), or failure, which implies that the conditions of the theorem were not met and $T$ is not close to $M$ in that $\|R\| \geq 1 /\|G\|$. A model can thus be conclusively invalidated, provided that a mechanism for implementing a closed-loop policy "live" on the true market exists. This is the value of a true economic laboratory, where experiments can be conducted and observed, even without perfect control over the internal variables or outside influences of the market.

\section{INDUSTRIAL ORGANIZATION}

\section{A. Profit Maximizing Dynamics}

Now, consider a market, $\mathcal{M}$, of $N$ products. Without loss of generality, give these products an arbitrary order and integer label so that $\mathcal{M}=\{1,2, \ldots, n\}$. Let $p \in \mathbb{R}^{N}$ be the vector of (non-negative) prices for these $N$ products, and let $q: \mathbb{R}^{N} \rightarrow \mathbb{R}^{N}$ be the (non-negative) demand for these products at prices $p$.

We now consider a firm, $F$ to be a subset of the $N$ products in the market, $F \in 2^{\mathcal{M}}$. This implies that the firm controls the production and distribution of the products assigned to it. Most importantly for our analysis, since we consider a Bertrand market model, this implies that the firm may set the prices of the $n=|F|$ products assigned to it.

We suppose that the products of the market are partitioned between $m$ firms. This implies that no two firms control the same product, $F_{i} \bigcap F_{j}=\emptyset \quad \forall i \neq j$, and that the union of all products assigned to the $m$ firms compose the entire market, $\bigcup_{i=1}^{m} F_{i}=\mathcal{M}$.

Let $c_{j}\left(q_{j}\right), j=1, \ldots, N$ be the cost of production of $q_{j}$ units of product $j$. The profit of the $i^{\text {th }}$ firm then is given by

$$
\pi_{i}=\sum_{j \in F_{i}}\left[q_{j}(p) p_{j}-c_{j}\left(q_{j}(p)\right)\right]
$$

A profit-maximizing firm under the Bertrand model of market behavior will tend to change its prices to maximize its short-term profit [7]. We model this behavior by assuming that the firm will evolve the prices of its products in the direction of maximally improving its profits. That is, if product $j$ belongs to firm $i$, then we expect the firm to evolve the price of product $j$ as

$$
\frac{d p_{j}(t)}{d t}=\left.\frac{\partial \pi_{i}(p)}{\partial p_{j}}\right|_{p(t)}
$$

where $p(t)$ is the pricing vector for the entire market at time $t$.

Notice that these dynamics suggest that if the partial derivative of profits is negative with respect to the price of product $j$, that the firm should decrease the price of product $j$. This is in the direction of improving profits. Likewise, if the partial derivative were positive, the firm would increase the price of product $j$ to improve profits. When the partial derivative is zero, the motivation is to hold the price at this locally profit-maximizing position.
Reordering the $N$ market products so that each firm's products are grouped together, and letting $n_{i}$ be the number of products controlled by firm $i$, we then can partition the pricing vector into components associated with each firm. If every firm in the market is assumed to be profit maximizing, this yields the following market dynamics:

$$
\left[\begin{array}{c}
\dot{p}_{1}(t) \\
\vdots \\
\dot{p}_{n_{1}}(t) \\
\frac{\dot{p}_{n_{1}+1}(t)}{\vdots} \\
\dot{p}_{n_{1}+n_{2}}(t) \\
\frac{\left(\partial \pi_{1} / \partial p_{n_{1}}\right)(p(t))}{\dot{p}_{\sum_{i=1}^{m-1} n_{i}+1}(t)} \\
\vdots \\
\dot{p}_{\sum_{N}}(t)
\end{array}\right]=\left[\begin{array}{c}
\left(\partial \pi_{1} / \partial p_{1}\right)(p(t)) \\
\frac{\left(\partial \pi_{2} / \partial p_{n_{1}+1}\right)(p(t))}{\vdots} \\
\frac{\left(\partial \pi_{2} / \partial p_{n_{1}+n_{2}}\right)(p(t))}{\vdots} \\
\frac{\left(\partial \pi_{m} / \partial p_{\sum_{i=1}^{m-1} n_{i}+1}\right)(p(t))}{\vdots} \\
\left(\partial \pi_{m} / \partial p_{N}\right)(p(t))
\end{array}\right]
$$

where the dot notation $\dot{p}(t)$ is used to represent $d p(t) / d t$.

Notice that if the market system (3) has an equilibrium, such a pricing vector $p_{e q}$ would represent prices from which no firm can improve its profits by unilaterally changing the prices over which it has control. Under certain technical conditions such an equilibrium can be shown to exist. Moreover, this equilibrium can often be shown to be asymptotically stable, in the sense that any pricing vector $p(0)$ will converge to the equilibrium $p_{e q}$ as $t \rightarrow \infty$.

\section{B. The Firm as a Coalition}

Under the assumption that the market dynamics are stabilizing, we expect price perturbations to re-equilibrate. In this context, it is convenient to simplify the problem by only considering the profits of the firms at equilibrium. These profits define a payoff function reminiscent of those used to define coalition games.

Let $v\left(F_{i}\right)=\left.\pi_{i}\right|_{p=p_{e q}}$ be the payoff or profit of firm $i$ at the market equilibrium prices $p_{e q}$. In this way the firm may be thought of as a coalition of $n_{i}$ players in an $N$-player cooperative game. Each player is a one-product company that completely manages the production, distribution, and pricing decisions for its product. The firm, then, is a confederacy of these one-product companies that works together to maximize their combined profits or payoffs.

The theory of coalition games studies the behavior of such coalitions once the payoff function is defined for every possible coalition [1], [5]. The idea is that any given coalition $F_{i}$ yields a well-defined payoff $v\left(F_{i}\right)$, and then a number of questions can be explored regarding how to distribute the payoff among the members of the coalition, etc.

Our situation is different because the payoff to a given firm doesn't just depend on the products it controls, but 
also on the market structure of the products outside the firm. For example, consider a 10-product market and a three product firm in the market. The payoff to the firm does not just depend on the prices of the three products it controls, but also on the prices of the other seven products. The profit-maximizing equilibrium prices of these other seven products, however, may be set differently depending on whether they belong to a single firm or whether they are controlled by seven different companies. Thus, the payoff to the three-product firm depends on the entire market structure.

Coalition game theory addresses such situations by considering partition systems and restricted games. For our purposes, it is sufficient to partition the $N$ products of $\mathcal{M}$ into $m$ firms and then assume that this structure is fixed outside of the particular firm that we are studying. This enables us to work with a well defined payoff function induced by the profit-maximizing dynamics of firms within the market without eliminating the multiple-coalition (i.e. multiple firm) cases of interest.

\section{Value of Cooperation}

To quantify the value of organizing a group of oneproduct companies into a single firm, we need to compare the profits the firm receives if it sets its prices as if each of its products were independent companies with those it realizes by fully capitalizing on cooperation between the products. More precisely, let $p_{e q}$ be the profit-maximizing equilibrium prices for the given market structure. In contrast, consider the new profit maximizing equilibrium prices achieved without cooperation if $F_{i}$ were divided into its constituent one-product companies and each independently optimized their prices. Let this second set of equilibrium prices serve as a basis for comparison, or reference, and be denoted $p_{\text {ref }}$. The relative value of cooperation (VC) of a given firm $F_{i}$ in market $\mathcal{M}$ with structure $S=$ $F_{1}, F_{2}, \ldots, F_{m}$ is then given by

$$
\mathrm{RVC}_{\text {ref }}\left(F_{i}, S\right)=\frac{\left.\pi_{i}\right|_{p_{\text {eq }}}-\left.\pi_{i}\right|_{p_{\text {ref }}}}{\left.\pi_{i}\right|_{p_{\text {eq }}}}
$$

This measure is interpreted as the percentage of profits due to cooperation within the organization. It is bounded between zero and one, and it facilitates direct comparison between firms of different sizes.

Sometimes we may be interested in measuring the value of cooperation between structures other than the current market structure and the reference structure. This could be the case when considering mergers between firms, or when management is considering selling off a piece of the firm. In such cases it is easy to extend the definitions of RVC by simply replacing the equilibrium and reference prices with the equilibrated profit-maximizing prices of the two market structures being compared.

It is instructive to contrast the RVC with other measures used to characterize cooperative games. Hart and MasColell [3] defined a measure, called the potential, $P$, that computes the expected normalized worth of the game i.e. the per-capita potential, $P / N$, equals the average per-capita worth $(1 / m) \sum_{i}\left(\pi_{i}\right) /\left(\left|F_{i}\right|\right)$. Given a market structure, this measure characterizes the expected profit of an averagesized firm (where size is measured with respect to the number of products the firm controls) in the market, even if such a firm does not actually exist.

Moreover, the potential has been connected to another measure, called the Shapley value, $\Phi_{j}$, which yields the marginal contribution of each product in the market [6]. This measure characterizes how the payoff of a coalition should be divided between members of the team. In both cases, the potential and Shapley value do not suggest anything about the intrinsic benefit of forming coalitions in the first place.

The Relative Value of Cooperation, RVC, on the other hand, captures the natural significance for organizing production into multi-product firms. Nevertheless, this measure does not yield any information about how the profit of a firm should be efficiently invested into each of the firm's constituent production lines. Thus, this measure is inherently different from the potential or shapely value since these focus more on the value of a member of a coalition to the group, rather than the value of the coalition as a whole.

\section{Example}

To illustrate the point, consider a two product economy with linear demand given by

$$
\left[\begin{array}{l}
q_{1}(t) \\
q_{2}(t)
\end{array}\right]=\left[\begin{array}{cc}
-3.5 & -1 \\
-3 & -2
\end{array}\right]\left[\begin{array}{l}
p_{1}(t) \\
p_{2}(t)
\end{array}\right]+\left[\begin{array}{l}
100 \\
100
\end{array}\right]
$$

Suppose that the unit production cost of each product is $c_{1}=10, c_{2}=10$. If we consider a market structure where each product is produced by an independent company, the profit function for each company becomes

$$
\begin{aligned}
\pi_{1}(t) & =q_{1}(t)\left(p_{1}(t)-c_{1}\right) \\
& =-3.5 p_{1}^{2}-p_{1} p_{2}+135 p_{1}+10 p_{2}-1000 \\
\pi_{2}(t) & =q_{2}(t)\left(p_{2}(t)-c_{2}\right) \\
& =-2 p_{2}^{2}-3 p_{1} p_{2}+30 p_{1}+120 p_{2}-1000
\end{aligned}
$$

Taking the partial derivatives of each profit function with respect to the appropriate pricing variable, we find the profit-maximizing market dynamics to be:

$$
\left[\begin{array}{l}
\frac{d p_{1}(t)}{d t} \\
\frac{d p_{2}(t)}{d t}
\end{array}\right]=\left[\begin{array}{ll}
-7 & -1 \\
-3 & -4
\end{array}\right]\left[\begin{array}{l}
p_{1}(t) \\
p_{2}(t)
\end{array}\right]+\left[\begin{array}{l}
135 \\
120
\end{array}\right]
$$

Figure 5 shows how the two-firm dynamics drive an initial pricing vector to a profit-maximizing equilibrium. This equilibrium price is

$$
p_{\text {ref }}=\left[\begin{array}{l}
16.8 \\
17.4
\end{array}\right]
$$

and the associated equilibrated profits are $\pi_{1}=161.84$, $\pi_{2}=109.52$. 


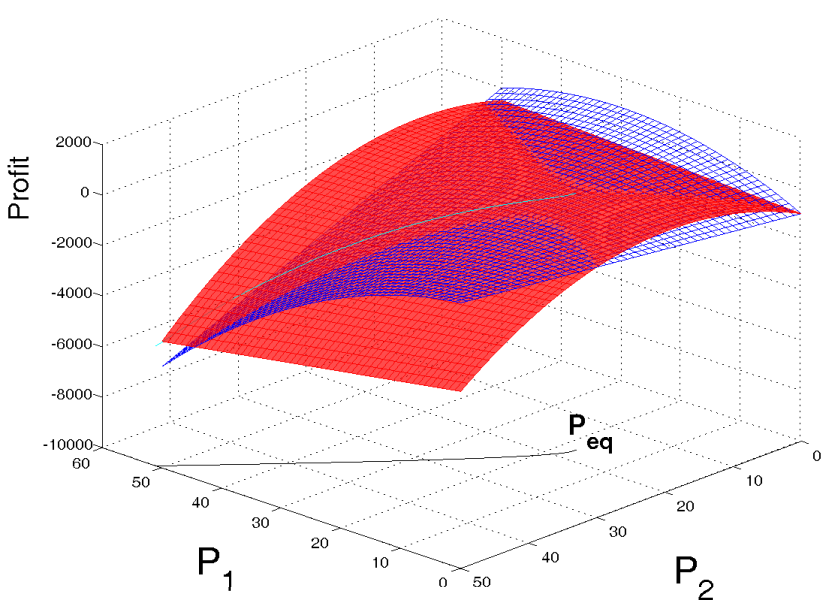

Fig. 5. Two firm price trajectory and profit function

Now, consider a market structure where both products are controlled by the a same firm. In this case, the firm's profit function becomes

$$
\begin{aligned}
\pi(t) & =q_{1}(t)\left(p_{1}(t)-c_{1}\right)+q_{2}(t)\left(p_{2}(t)-c_{2}\right) \\
& =-3.5 p_{1}^{2}+165 p_{1}-4 p_{1} p_{2}+130 p_{2}-2 p_{2}^{2}-2000
\end{aligned}
$$

With this market structure, the firm adjusts the prices of both products to optimize the same objective. These new dynamics become:

$$
\left[\begin{array}{l}
\frac{d p_{1}(t)}{d t} \\
\frac{d p_{2}(t)}{d t}
\end{array}\right]=\left[\begin{array}{ll}
-7 & -4 \\
-4 & -4
\end{array}\right]\left[\begin{array}{l}
p_{1}(t) \\
p_{2}(t)
\end{array}\right]+\left[\begin{array}{l}
165 \\
130
\end{array}\right]
$$

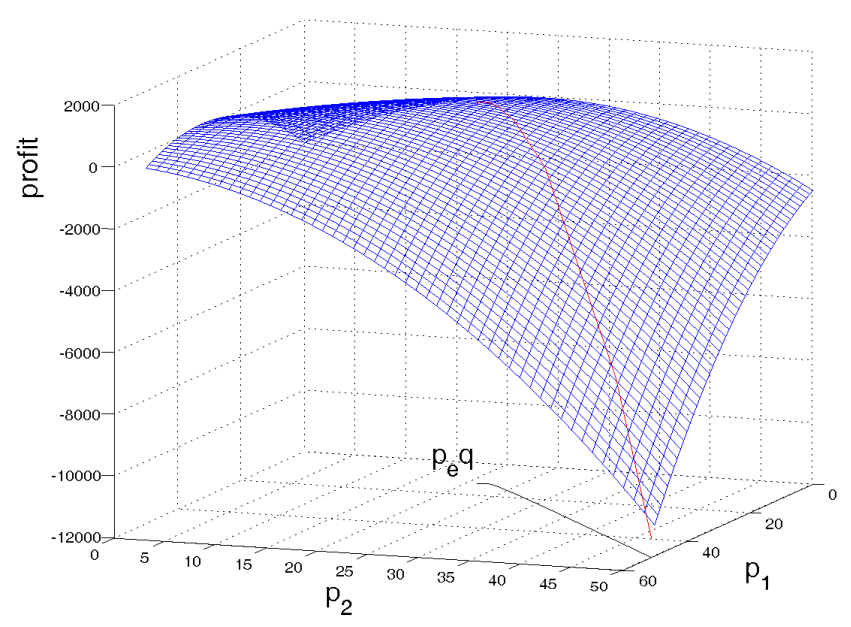

Fig. 6. One firm price trajectory and profit function

Figure 6 shows how the single-firm dynamics drive an initial pricing vector to a profit-maximizing equilibrium. The new equilibrium price is given by

$$
p_{e q}=\left[\begin{array}{l}
11.67 \\
20.83
\end{array}\right] \text {. }
$$

and the associated equilibrated profits are $\pi_{e q}=316.667$. The relative value of cooperation in this example thus becomes

$$
R V C=0.1431
$$

This suggests that in this market, just under $15 \%$ of the profits of the two-product firm are due to cooperation.

\section{CONCLUSION}

This paper explored two applications of dynamic analysis in economic systems. The first considered a firm to be an operator in feedback with its associated market. The firm is characterized by its pricing policy mapping sales data into prices. We discussed problems associated with the verification of such policies, and showed how robustness results from the small gain theorem suggest that a true retail laboratory could be feasible.

The second problem quantified measures to calibrate the value of cooperation within a specific firm in a given market. The idea is to assume profit-maximizing dynamics among the firms within the market and compare equilibrium profits in two different scenarios. The first scenario considers the firm as it is, as a single economic entity with a unified objective and exhibiting full cooperation between its various economic units. The second scenario considers splitting the firm into its constituent economic units and computing market equilibrium prices if these units maximized their individual profits. Normalizing the difference between the cooperative profits of the first scenario and the aggregate profits of the independent units of the second scenario define a measure we call the Relative Value of Cooperation, RVC, of the firm in its current market environment. This measure reveals the percentage of profits derived from cooperation within the firm. Quantifying the value of cooperation is a first step in understanding how firms exert market power in their respective environments. This information is important for both managers, who hope to leverage the information to better lead their organizations, and regulators, who monitor the impact of corporate decisions on social welfare [2].

\section{REFERENCES}

[1] J.M. Bilbao. Cooperative games on combinatorial structures. Kluwer Academic Publishers, Dordrecht, 2000.

[2] F. Fisher. Diagnosing monopoly. Quarterly Review of Economics and Business, 19, 1979.

[3] Sergiu Hart and Andreu Mas-Colell. Potential, value, and consistency. Econometrica, 57(3):589-614, 1989.

[4] D.A. Kendrick. Stochastic control for economic models: Past, present, and the paths ahead. Ninth International Conference of the Society of Computational Economics, Keynote Address, 2002.

[5] P. Morris. Introduction to Game Theory (Universitext). SpringerVerlag, 1994.

[6] L.S. Shapley. A value for $n$-person games. In H.W. Kuhn and A.W. Tucker, editors, Contributions to the Theory of Games, Vol. II, volume 28 of Annals of Mathematics Studies, pages 307-317. Princeton University Press, Princeton, NJ, 1953.

[7] O. Shy. Industrial Organization: Theory and Applications. The MIT Press, 1996.

[8] K. Talluri and G. Van Ryzin. The Theory and Practice of Revenue Management. Kluwer, Boston, 2004.

[9] H. Varian. What use is economic theory? Prepared for the conference, 'Is Economics Becoming a Hard Science', Paris, France, 1992. 